\title{
Seroreactive Donor Demography, Notification and Counseling in Present Era of Information Technology
}

\author{
Trivedi $\mathrm{PK}^{1 *}$, Dighe $\mathrm{MP}^{2}$, Kothari $\mathrm{F}^{3}$ \\ 1. $3^{\text {rd }}$ year Resident, Immunohaematology and blood transfusion, SSG Hospital, Baroda, Gujarat \\ 2. HOD, Immunohaematology and blood transfusion, SSG Hospital, Baroda, Gujarat \\ 3. Associate Professor, Immunohaematology and blood transfusion, SSG Hospital, Baroda, Gujarat
}

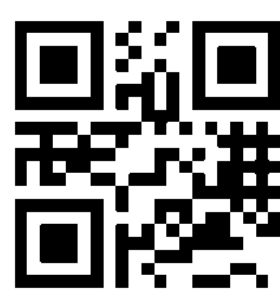

Abstract:

Background: As blood transfusion is associated with risk of transmitting transfusion transmitted infections (TTI), another tool to prevent it is to inform, notify and counsel the donors about their seroreactive status at the blood centre. Methods: A retrospective study was carried out in department of IHBT, at BMC from January 2017 to October 2019. The reactive donors were informed by the blood bank counselor about an abnormal test result with an advice to report to the blood bank for one to one counseling and repeat testing, as well as for referral to the respective department or Integrated counseling and testing centre or sexually transmitted disease clinics of the hospital for further management by means of telephonic calls, letters and personal interrogation. The response rate of TTI reactive donors after notification was evaluated. Results: There were total 41,114 blood donation over the period of almost 3 years out of which 617 were reactive donors (1.5\%) with median age of 31.5 years and female to male proportion of 7:610. History of tattooing for more than 1 year was present in 155 donors. Amongst reactive donors 336 were voluntary while 281 were replacement donors. Seropositivity for HIV, $\mathrm{HBV}$ and Syphilis was more in replacement donor while HCV was more in voluntary donors. HBV, HCV, Syphilis and HIV were $316(0.87 \%), 136(0.33 \%), 69(0.17 \%)$ and $56(0.13 \%)$ respectively in reference to total population. There were 4 cases of co-infection 2 for HIV+HBV, 1 for HBV + Syphilis and 1 for HIV+HCV. All donors were contacted telephonically. Inaccurate donor demographics (wrong address and phone number) resulted in our inability to notify $6(0.97 \%)$ cases. Out of 56 HIV reactive donors 26 were voluntary and 30 were replacement donors and response rate for post donation counseling was 98\%, 4 HIV donors were previously registered at ART centre and 4 became non reactive so they were again followed up after 3 months. Conclusion: A large majority of the notified donors in our study contacted their health care provider when given clear instructions to do so. Stringent notification data on HBV, HCV and Syphilis reactive donors is required. Thorough tracking system for follow up of reactive donors is required.

Key words: HBV, HIV, Seroreactive, Syphilis,

\section{*Corresponding Author}

Dr. Para K. Trivedi

E-mail id: paraktrivedi@gmail.com

Copyright: This is an open-access article distributed under the terms of the Creative Commons Attribution license which permits unrestricted use, distribution, and reproduction in any medium for non-commercial use (Non Commercial, or CC-BY-NC) provided the original author and source are credited.

\section{INTRODUCTION}

With every unit of blood, there is $1 \%$ chance of transfusion-associated problems including transfusion transmitted infections [TTI]. ${ }^{1}$ Blood transfusion is associated with the risk of transmitting transfusion transmissible infections (TTI) even after the thorough mandatory TTI screening of blood units. Donor cycle starts with donor recruitment and ends with donor retention and recall or with notification of seroreactive donors. $^{2}$ Notifying and counselling the TTI seroreactive donors along with donor education is an important and efficient method of curtailing TTI. ${ }^{3}$ Post test counselling and notification of positive donors is required for the health of donor and his family, prevention of diseases, improving blood bank economy by avoiding wastage of blood and reducing exposure to HCW. Nonnotified donors continue to donate blood leading to wastage or breach of patient safety if they get inadvertently transfused. Problems in notification include donor anxiety as every donor reacts in a different manner. ${ }^{2}$ In India, disclosure of viral TTI reactivity to blood donor was not permitted until December 2004, after that NBTC formed a strategy for notification.4 Blood banks obtain written consent at the time of donation from donors for informing about reactive test results. Reactive donors are intimated telephonically and by post for one to one counselling and repeat sampling. ${ }^{5}$ Response rate to notification is often poor. Previous studies show that donors who

Volume-9 | Issue-2 | $2020 \mid 21$ 
were notified regarding their results neither responded at all nor followed up for the counselling and some of them continue to donate blood despite being notified. ${ }^{6}$ The present study determines the response of various TTI reactive donors for post donation counselling after notification and their persistence in society as reactive donors.

\section{MATERIALS AND METHODS}

A retrospective study was carried out in blood bank, department of immuno haematology and blood transfusion, at a tertiary care government hospital of middle Gujarat from January 2017 to October 2019. History of IV drug use, sexual history, tattooing, previous blood transfusion, liver disease etc were asked and ruled out in pre donation counselling. The donated blood was tested for TTIs like Human Immunodeficiency Virus (HIV), Hepatitis B Virus (HBV), Hepatitis $\mathrm{C}$ Virus (HCV), Syphilis and Malaria.

Methods used for screening:

$\begin{array}{ll}\text { HIV } & \text { ELISA (Enzaids / Erba) } \\ \text { HBsAg } & \text { ELISA (Erba / Merilisa) } \\ \text { Anti-HCV } & \text { ELISA (Erba / Merilisa) } \\ \text { Syphilis } & \text { Rapid Plasma Reagent test (Reckon) } \\ \text { Malaria } & \text { peripheral smear (thick smear) }\end{array}$

The reactive donors were informed by the blood bank counsellor about an abnormal test result with an advice to report to the blood bank for one to one counselling and repeat testing, as well as for referral to the respective department or Integrated counselling and testing centre or sexually transmitted disease clinics of the hospital for further management by means of telephonic calls and letters or sometimes reaching at the address given by the donor on consent form. Confidentiality was maintained at every step. The response rate of TTI reactive donors after notification was evaluated. The study was approved by the department of Immuno Haematology and Blood Transfusion, S.S.G. hospital and Medical College Baroda. The statistical method used here were mean, median, mode and percentage.

\section{RESULTS}

There was total 41,114 blood donation over the period of almost 3 years out of which 617 were reactive donors $(1.5 \%)$. The median age of reactive donors was 31.5 years. Out of 617 reactive donors 7 were female while rest of the 610 were male. History of tattooing for more than 1 year was present in about 155 donors. Out of 617 reactive donors 336 were voluntary where as 281 were replacement donors. Seropositivity for HIV, HBV and Syphilis was more in replacement donor while for $\mathrm{HCV}$ was more in voluntary donors. There were 4 cases of coinfection 2 for HIV + HBV and 1 for $\mathrm{HBV}+$ Syhilis and 1 for HIV + HCV. All donors were contacted telephonically. Inaccurate donor demographics (wrong address and phone number) resulted in our inability to notify 6 $(0.97 \%)$ cases. Out of 56 HIV reactive donors 26 were voluntary and 30 were replacement donors. Out of which 4 HIV donors were previously registered at ART centre and 4 became non reactive so they were again followed up after 3 months. The response rate for overall post donation notification was $99 \%$. The response rate was highest in donors reactive for HIV- 100\% (56/56) and syphilis 100\% (69/69) followed by HCV-98.53\% (134/136) and HBsAg- 98.87\% (352/356).

Table 1: Donor Notification And Response Rate

\begin{tabular}{|l|l|l|l|l|}
\hline & HIV & HBV & HCV & Syphilis \\
\hline Contacted & 56 & 352 & 134 & 69 \\
\hline Responded & 56 & 352 & 134 & 69 \\
\hline Total & 56 & 356 & 136 & 69 \\
\hline
\end{tabular}

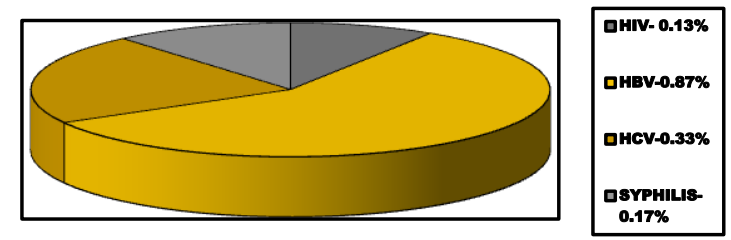

Figure 1: Percentage of Seroreactivity for Different Infections

\section{DISCUSSION}

Notifying reactive donors is a very sensitive aspect as it has various psychological and social impacts. $^{7}$ Donor notification which was once nonexistent, has become one of most important topic in India as print and electronic media are constantly highlighting the demerits of not notifying donors. ${ }^{2}$ Response of reactive donors to seek confirmation and treatment is a direct reflection of their knowledge and attitudes towards TTIs. Every donor reacts in a different manner, some get angry, some face a period of denial and others have complete nervous breakdown.The important goals of the notification process are to make sure that donors are informed about their test results and that donors whose test results make them ineligible for future donation understand their deferral status. Our study indicate that the notification process does not always achieve these goals as in our study only 611 were contacted and remaining $6(0.97 \%)$ could not be contacted (phone switch off, not responding, or not available) Incomplete demographic details given by donors was the major limiting factor in communicating. This high percentage of false information may be due to unawareness towards TTIs and not understanding the importance of giving their 
correct phone numbers and addresses. There is also possibility of known reactive status and act of purposely giving wrong phone numbers and address in attempt to conceal their identity. Donors which could not be contacted poses a high risk on society as they keep on donating blood. Low response rate in the donors were due to poor health care knowledge and poor understanding of screening results. Response rate was higher for HIV and Syphilis reactive donors, higher response rate for HIV was also noticed in other studies. ${ }^{9-11}$ Higher response rates for HIV reactive donors might be due to the higher awareness and fear of HIV/AIDS among the general population. ${ }^{11}$ Notification to donors and post-donation counseling has proven to be beneficial to the blood donors, blood center and community at large, as it reduces the risk of spreading of diseases. All donors must be screened for high risks behaviors, as TTIs can exist as asymptomatic diseases in the hosts and the acquisition of the infections in the healthy blood donor population can be a serious threat to the safety of the collected blood donations. Disclosure and counseling process immensely benefits the donor, as early diagnosis helps them to manage and start treatment, if required. All necessary preventive interventions can be initiated for safety of the donor and his/her family members. ${ }^{8}$

\section{CONCLUSION}

Donor notification and post-donation counselling are an essential aspect of the blood bank that entails provision of information on serological status, assess the impact of test results on the donor and finally referral for medical care. A large majority of the notified donors in our study contacted their health care provider when given clear instructions to do so. Stringent notification data on HBV,HCV and Syphilis reactive donors is required. Thorough tracking system for follow up of reactive donors is required.There is needed to create more awareness among the donors to achieve the Goal of "Safe Blood starts with me". Strategies need to be framed for spreading the importance of self deferral. Donor registration should be clear and understandable and demographic details should be verified with valid government identification proof for the ease in contacting donors. Predonation counseling is a very important aspect as donor education and motivation about the importance of TTI status if known and any high risk behavior can help them from self excluding from donation and to clarify myths and misconceptions.

Conflicts of interest: Declared, authors have no conflict of interest

Funding/Financial support: None

\section{REFERENCES}

1. Chandra T, et al. Prevalence of transfusion transmitted infections in blood donors an Indian experience. Trop Doct 2009. 39:152154.

2. Chandrashekar S, Kantharaj A. Blood donor notification: Boon for the community, bane for blood donors, and blood centers? Glob J Transfus Med. 2018;3:6-12.

3. Bianco C, Kessler D. Donor notication and counseling. Management of blood donors with positive test results. VoxSang. 1994;67(3):255-9.

4. National AIDS Control Organisation. National Blood Policy of India. Available at: http://www.unpan1.unorg/intradoc/groups/pu blic/documents/.pdf. Accessed on 3 March 2019.

5. Dontula $\mathrm{S}$, Mathur A, Kamaladoss $\mathrm{T}$, Adimurthy S, Jagannathan L. Donor disclosure - a donor's right and blood bank's responsibility. Transf Alter Transf Med. 2012;12:44-50.

6. Patel SG, Patel JN, Patel AC, Raja KA, Dobariya GH, Pandya AN. Blood Donor notification and counseling of reactive test result in Blood Bank of South Gujarat: A better approach to prevent reactive donors from donating blood again. Glob J Transfus Med. 2016;1:57-60.

7. Kumari S. Reactive donor notification and counseling: reveals concealed risk factors. Indian J Soc Psychiatry. 2017;33:38-43.

8. Kaur G, Kaur P, Basu S, Kaur R, Sharma S. Donor notification and counseling Experience and challenges. TransfusApher Sci. 2013;49:291-4.

9. Handa A et al. Int J Community Med Public Health. 2019 Jul;6(7):2794-2797

10. Kumari AB, Deepa S, Venkatesha D. Blood transfusions: are they lifesaving or transfusing infections?. Online J Health Allied Sci. 2011;10(2).

11. Kaur G, Kaur P, Basu S, Kaur R, Sharma S. Donor notification and counsellingexperience and challenges. Transfusion Apheresis Sci. 2013;49(2):291-4. 